Case Report

\title{
Amelogenesis Imperfecta, HypoplasticType with Multiple Impacted Teeth - A Case Report
}

\author{
Sushmini Hegde ${ }^{1}$, Hemcle Shalma Ganesan ${ }^{1,}$, , Karidhi Sudesh Suraj ${ }^{2}$ \\ ${ }^{1}$ Department of Oral Medicine and Radiology, the Oxford Dental College and Hospital, Bengaluru, India \\ ${ }^{2}$ Department of Prosthodontics and Crown and Bridge, the Oxford Dental College and Hospital, Bengaluru, India \\ Email address: \\ shalmajoy@gmail.com (H. S. Ganesan) \\ ${ }^{*}$ Corresponding author
}

\section{To cite this article:}

Sushmini Hegde, Hemcle Shalma Ganesan, Karidhi Sudesh Suraj. Amelogenesis Imperfecta, Hypoplastic Type with Multiple Impacted Teeth A Case Report. International Journal of Dental Medicine. Vol. 4, No. 1, 2018, pp. 27-30. doi: 10.11648/j.ijdm.20180401.16

Received: July 23, 2018; Accepted: August 9, 2018; Published: September 5, 2018

\begin{abstract}
Amelogenesis imperfecta (AI) is a developmental disorder of genomic origin associated with structural enamel formation. Hereditary brown enamel, hereditary enamel dysplasia, hereditary brown opalescent teeth are the other terminologies used for AI. The prevalence varies from 1:700 to 1:14 000, according to the populations studied. AI affects the entire ectodermal component and the enamel may be hypoplastic, hypomineralized or both and affected teeth may be esthetically and functionally compromised, discolored, sensitive or prone to disintegration, leading to loss of occlusal vertical dimensions and very poor aesthetics. Although AI is considered as a single disease entity, it actually represents a group of heterogenous conditions, with diverse structural defects of enamel resulting in a range of clinical phenotypes. It is characterized by clinical and genetic heterogeneity in the absence of systemic abnormalities or diseases. AI had been found to be associated with non-enamel anomalies such as delayed eruption, crown resorption, congenitally missing teeth, pulpal calcifications, dental follicular hamartomas, and gingival hyperplasia. Mutations in the amelogenin, enamelin, and kallikrein-4 genes have been demonstrated to different types of AI.Restoration of the dentition poses a great challenge when all the teeth are severely affected. The treatment opportunities for these patients may help in refining their lifestyle. This case report reviews a holistic multidisciplinary treatment approach for the oral rehabilitation of patient with hypoplastic AI with multiple impacted teeth.
\end{abstract}

Keywords: Amelogenesis Imperfecta, Hypoplastic, Impacted Teeth

\section{Introduction}

$\mathrm{AI}$ is a term for a clinically and genetically heterogenous group of conditions that affect the dental enamel, occasionally in conjunction with other dental, oral and extraoral tissues. AI is typically characterized by generalized enamel defects in both primary and permanent dentition. It might also be associated with morphological or biochemical changes elsewhere in the body.[1]

The prevalence of this condition has been expected to range from 1 in 718 to 1 in 14,000, depending on the population studies. Hypoplastic AI represents $60-73 \%$ of all cases, Hypomaturation AI represents $20-40 \%$ and Hypocalcification AI represents 7\%.[2]

AI has six times more tendency than unaffected people to have impacted permanent teeth and is often associated with anomalies such as follicular cysts.[3] Diagnosis involves exclusion of extrinsic environmental or other factors, establishment of a likely inheritance pattern, and recognition of phenotype and correlation with the dates of tooth formation to exclude a chronological developmental disturbance.[4] The enamel may be hypoplastic, hypomineralized or both, and teeth affected may be discoloured, sensitive or prone to disintegration either post-eruption (post-eruptive breakdown) or pre-eruption (idiopathic resorption).[5]

\section{Case Report}

A 19 year old male patient presented with a chief complaint of yellowish discoloration of teeth and unsatisfactory dental 
appearance since childhood. There was no associated pain but difficulty in chewing reported. He resided in a non fluoridated area since his birth. His maternal and post natal history was not significant. Familial history revealed consanguineous marriage. No other family member had similar dental problem.

General examination suggested him to be in a good state of health. Extraoral examination revealed a short and broad face (Figure1) with a concave profile and mid facial hypoplasia noted. (Figure2)

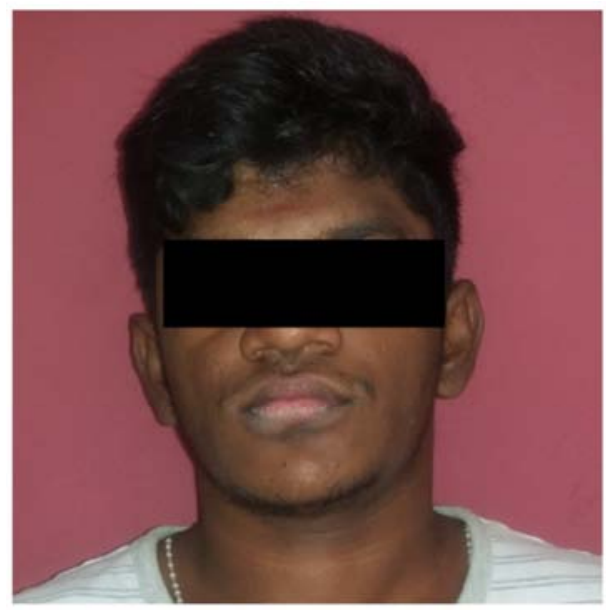

Figure 1.Short and broad face.

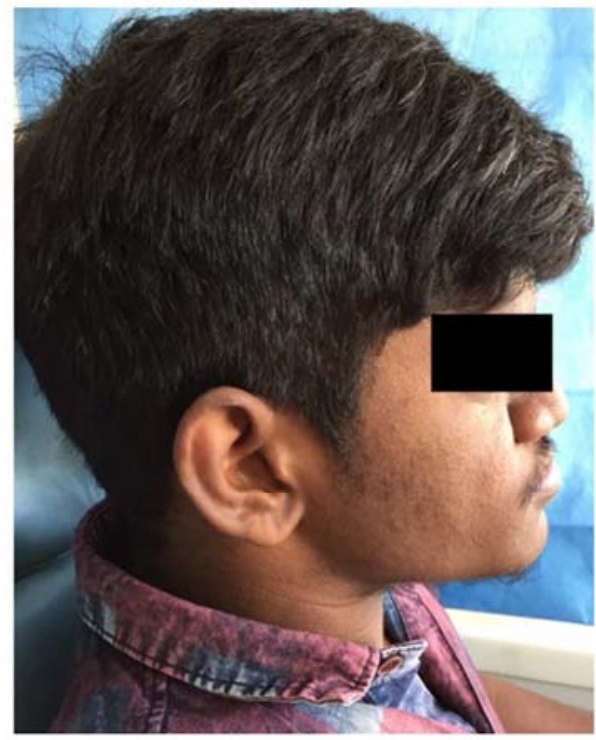

Figure 2.Concave profile.

His hair and nails were normal and patient had saddle nose. Intraoral examination revealed multiple unerupted permanent teeth. Teeth present were $15,16,22,24,25,26,31,32,33,74$, $37,41,42,43,44$. The erupted dentition had yellowish brown discolored hypoplastic enamel with no proximal contact. Enamel appeared thin and smooth over the entire crown. There was partial anodontia, one retained deciduous tooth, anterior and posterior open bite and a loss of vertical dimension. Severe attrition of teeth was present. (Figure 3, 4)

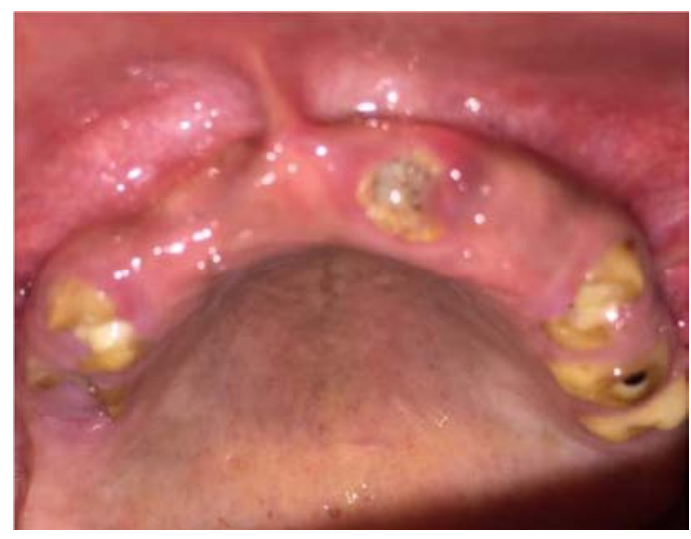

Figure 3.Attrition of upper teeth.

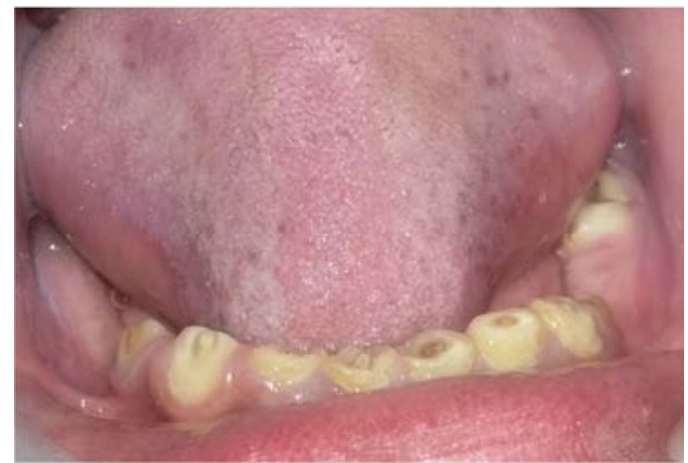

Figure4. Attrition of lower teeth.

Lower front side intraoral periapical (IOPA) radiograph showed teeth with short root, periapical radiolucency, external root resorption and exposed pulp horn due to attrition. (Figure: 5)Panoramic radiograph showed many teeth with stunted roots and malformed crown, multiple impacted permanent teeth and congenitally missing teeth. No peripheral enamel overlying the dentin could be appreciated in the radiographs, as the normal enamel-dentin contrast was absent. Multiple periapical radiolucencies were also seen, in relation to many attrited teeth. Few impacted teeth had an increased follicular space. (Figure: 6)



Figure 5. Intraoral periapical radiograph showing short roots with periapical radiolucency. 


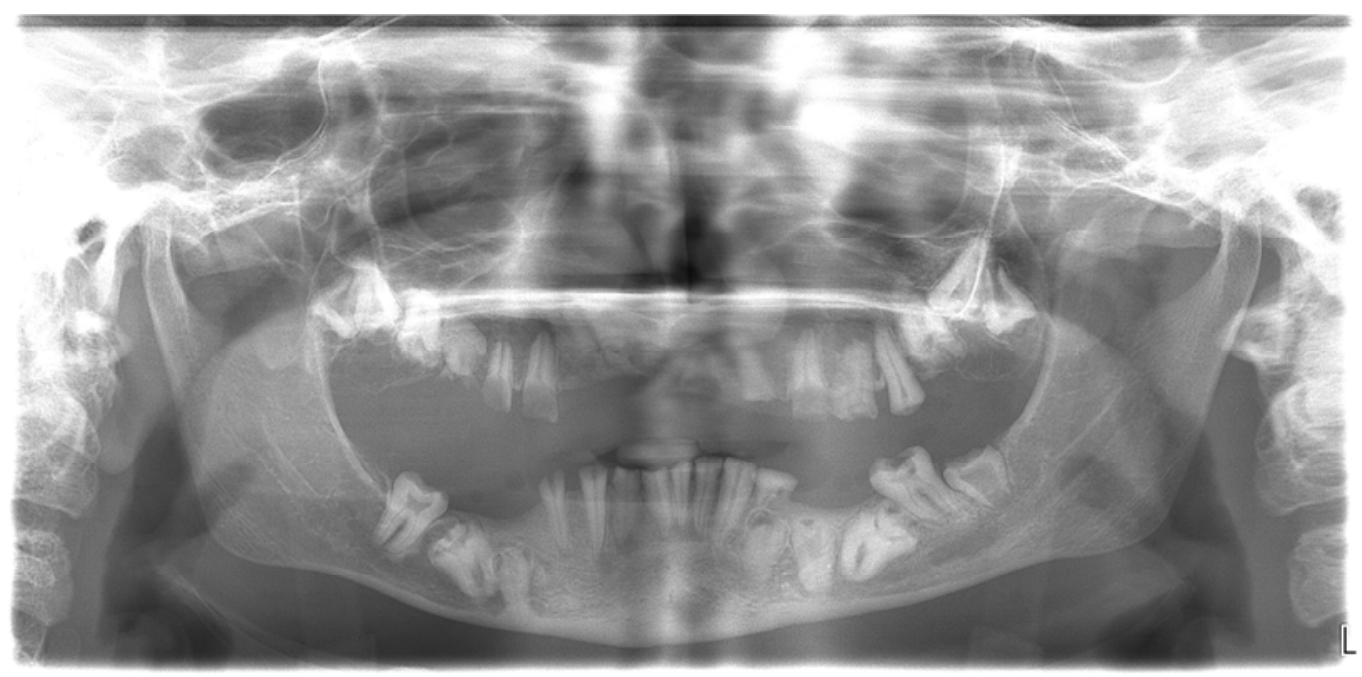

Figure 6.Panoramic radiograph.

On the basis of clinical and radiographic features, final diagnosis of hypoplastic AI with multiple impacted teeth was confirmed. Treatment options for full mouth oral rehabilitation includes extraction of impacted teeth, replacement of missing teeth and protecting the existing dentition with crowns and bridges. (Figure: 7)

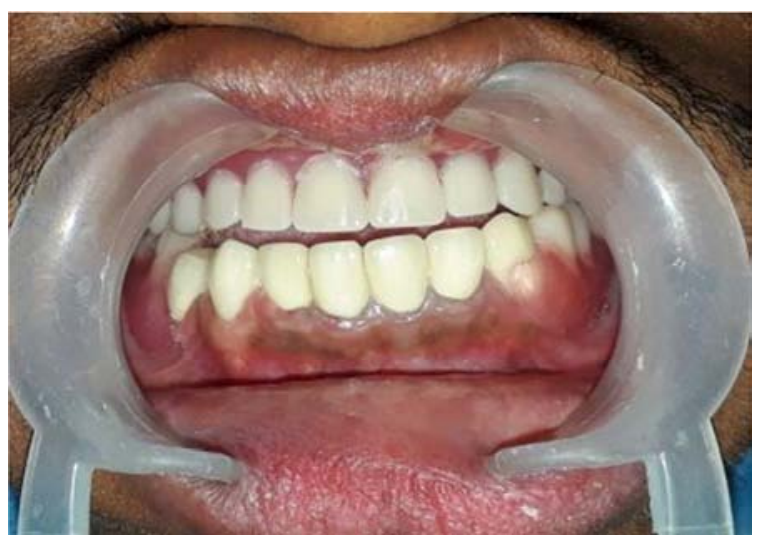

Figure7. After complete prosthetic rehabilitation.

\section{Discussion}

AI is a developmental, often inherited disorder, affecting dental enamel. It usually occurs in the absence of systemic features and comprises of diverse phenotypic entities. Compared with Dentinogenesis imperfecta the patient does not usually complaint of sensitivity since the dentin is intact. This is in accordance with our case where patient was asymptomatic except for unpleasant appearance since childhood.

Although there are several methods to determine the type of AI using combinations of clinical, radiographic, histological, and genetic criteria, clinicians rely on clinical and radiographic findings when diagnosing and planning treatment for patients with AI. The clinical features distinguish the hypoplastic and hypocalcified types. In the hypoplastic forms, the enamel does not develop to its normal thickness. In the hypocalcified forms, the enamel thickness on the newly erupted teeth closely approaches that of normal teeth, but the enamel is soft, friable, and can easily be removed from dentin. In contrast to hypoplastic types, the hypomaturation types develop enamel of normal thickness. The hypomaturation forms differ from hypocalcification in that the enamel is harder, with a mottled opaque white to yellow-brown or red brown color, and tends to chip from the underlying dentin rather than wear away. [6-8]

AI has 6 times more tendency than unaffected people to have impaction of permanent teeth and associated anomalies such as follicular cysts.[3] Impaction can be attributed to the concurrent follicular enlargement, abnormality in molecular control of eruption process, space deficiency and dental alignment problems.[9]

The most substantial findings comprise extensive loss of tooth tissue, tooth sensitivity, excessive attrition leading to a short clinical crown, spacing in the anterior region of the dentition and normal or light proximal contacts in the posterior region. Abnormal tooth eruption, morbid root and coronal resorption, congenitally missing teeth, malocclusion, anterior open bites, pulpal calcification, hypercementosis, root malformation and taurodontism have been ordinarily repeated. Pitted enamel surfaces seen in AI can predispose to plaque accumulation and subsequent periodontal problems. However, orthodontic malocclusion like open contacts reduces the caries incidence. [10] AI is rarely associated with syndromes such as AI with taurodontism, tricho-dento osseous syndrome, AI with nephrocalcinosis and cone-rod dystrophy with AI. Tricho - dento osseous syndrome is an autosomal dominant condition characterized by splitting of the superficial layers of the nails, Kinky or tightly curled hair, bone sclerosis of long bones and skull base, zones of provisional calcification in the long bone, taurodontism, and enamel hypoplasia that occurs with hypomaturation/hypocalcification defects [11].

Koruyucu, et al. 2014 included 31 cases of AI in his study and found out that the main complaints of patients with AI 
were dissatisfaction with the appearances of their teeth, extreme dental sensitivity, other dental anomalies such as open bite and orthodontic problems. He concluded the study that the patients with AI may have a open bite, delayed tooth eruption, pathologic root resorption, pulpal calcifications, taurodontism, hypodontia, cross bite deep bite, prognathism, and retrognathism.[12] Most of the features in this particularcase report were similarto the study by Koruyucu et al.

Treatment planning for patients with AI is related to many factors, the age and socioeconomic status of the patient, the type and severity of the disorder and the intraoral situation. An interdisciplinary approach is necessary to evaluate, diagnose, and resolve aesthetic problems using a combination of periodontal, prosthodontic and restorative treatment It includes removal of surface stains, reducing sensitivity, maintaining vertical dimension of occlusion, and the esthetics with adhesive techniques/over dentures/porcelain - fused - to metal crowns/fixed partial dentures/full porcelain crowns/inlay/onlay restorations are used for better esthetics of the patients. Management of AI in the young adult using fixed prosthodontics is not a novel approach, but is possibly an underutilized one. The fixed prosthodontic treatment selected, albeit invasive, is more conservative than other considered alternatives. [13]

AI presents with problems of socialization, function and discomfort which may be managed by early vigorous intervention, both preventively and restoratively, with treatment continued throughout childhood and into adult life. Preventive aspects in the primary and mixed dentition include dietary advice, fluoride supplements, and oral hygiene instructions. Topical fluoride application can be done in the permanent dentition. [13]

\section{Conclusion}

$\mathrm{AI}$ is a serious problem that can result in reduced oral health-related quality of life. AI affects the psychology of the patient negatively due to aesthetic concerns. It should be promptly identified and treated and the existing dentition should be protected so that teeth can be conserved as much as possible. Patients should be counselled and motivated to maintain good oral hygiene. The dentist has to balance the decision for early intervention and long time survival of the restorations to prevent later problems.

\section{Summary}

This clinical report described the oral rehabilitation of a young adult patient affected by hypoplastic AI with multiple impacted teeth. The rehabilitation included removable partial denture in upper arch and fixed partial denture in lower archto eliminate tooth sensitivity, improve esthetics, and restore function.

\section{References}

[1] Backmon.B. Inherited enamel defects. Ciba Found symp. 1997; 205:175-182.

[2] Aldred M.J, Crawford P.J. AmelogenesisImperfecta; towards a new classification. Oral Dis.1995;1:2-5.

[3] Collins MA et al. Dental anomalies associated with amelogenesisimperfecta: A radiographic assessment. Oral Surg Oral Med Oral Pathol Oral RadiolEndod 1999; 88:358-64.

[4] Peter T, Titus S, Francis G. Full mouth rehabilitation of a case with amelogenesisimperfecta. SRM University Journal of Dental Sciences; 2010: 1(3).

[5] Shetty YB, Shetty A. Oral Rehabilitation of a Young Adult with AmelogenesisImperfecta: a case report. J Indian ProsthodontSoc; 2010; 10(4):240-245.

[6] WitkopJr CJ, Kuhlmann W, Sauk J. Autosomal recessive pigmented hypomaturationamelogenesisimperfecta. Report of a kindred. Oral Surg Oral Med Oral Pathol.1973;36:367-382.

[7] Sengün A, Özer F. Restoring function and esthetics in a patient with amelogenesisimperfecta: a case report. QuintessenceInt2002;33:199-204.

[8] Fischman SL, Fischman BC.

Hypoplasticamelogenesisimperfecta: report of case. J Am Dent Assoc1967;75:929-931Reddy S, Nisha V, Harish BN. Hypoplasticamelogenesisimperfecta with multiple impacted teeth - report of two cases. J ClinExp Dent. 2010; 2(4):e207-11.

[9] Moretti. A.B.S, Sakai.V.T, Oliveira.T.M Oral management of a child with mixed dentition affected by AmelogenesisImperfecta. Journal of Dentistry for Children. 2007; 74(2):157-160.

[10] Begum N, Bhandarkar GP, Kini R, Naik V, Rashmi K, D Souza LC. AmelogenesisImperfecta: A Series of Case Report. Int J Adv Health Sci 2015;2(1):17-21.

[11] Koruyucu M, Bayram M, Tuna EB, Gencay K, Seymen F. Clinical findings and long-term managements of patients with amelogenesisimperfecta. Eur J Dent 2014; 8:546-52.

[12] Mehta DN, Shah J, Thakkar B. AmelogenesisImperfecta: four case reports. J Nat SciBiol Med 2013 Jul-Dec;4(2):462-465. 